\title{
Thermal Conductivity Enhancement of Epoxy Resin Thermal Interfacial Materials Containing Ni Nanoparticle-Decorated Graphene Nanosheets
}

\author{
Lifei Chen, Lei An, Wei Yu, Huaqing Xie \\ College of Engineering, Shanghai Second Polytechnic University \\ Jinhai Road 2360, Pudong, Shanghai, China \\ lfchen@sspu.edu.cn
}

\section{Extended Abstract}

Graphene nanosheet (GNS) have large specific surface area, extraordinary physical properties [1], and strong interactions towards metals [2-4]. So it is possible that GNSs can be used as supporting materials for dispersing the metal nanoparticles directly on their surfaces. Because of GNS extraordinary thermal conductivity $(k)$ GNSs and its derivatives are important thermal conductive filler materials for polymer composites [5,6]. Si et al. has verified that the introduction of metai nanoparticles into the dispersion of graphene sheets impeded the formation of a stacked graphitic structure [7]. The existing of metal particles can decrease the possibility of GNS aggregation and to keep GNNs like other dispersions of nanomaterials with high aspect ratios.

In this paper, "mix-and-heat" method is applied to prepare Ni-GNSs [8]. The obtained product as thermal conductive fillers is used to prepare epoxy resin based TIMs. The epoxy resin based thermal interfacial materials (TIMs) with high thermal conductivity $(k)$ have been obtained by filling Ni nanoparticle-decorated graphene nanosheets (GNSs) as thermal conductive fillers. The $k$ of TIM samples is tested by using thermal conductivity meter (C-THERM TCI). The effect of thermal filler loading, the decoration quantity of Ni nanoparticles, and temperature on the thermal conductivity enhancement have been studied. Adding Ni-GNS to the epoxy resin results in high enhancement in $k$, with $k$ enhancement up to $231.6 \%$ observed for Ni-GNS loading at $5.0 \mathrm{wt} \%$ in epoxy resin as the quantity of Ni nanoparticles decorated on GNS is $1.5 \mathrm{~mol} \%$. The results showed that the $k$ enhancement of epoxy resin based TIMs increases with the thermal filler loading. The more decoration of Ni nanoparticles on the GNS surfaces, the higher thermal conductivity enhancement of epoxy resin based TIM is. It is proposed that the bigger Ni nanoparticles acting as "spacers" increase the distance between the graphene sheets more than the smaller ones. It is not easy for graphene sheets to form stacked graphitic structures and the high specific surface area as well as other unique properties exhibited by $2 \mathrm{D}$ graphene are retained. Furthermore, the larger particle size is desired to minimize the scattering of phonons because of low interfacial thermal barrier. The obvious enhancement of thermal properties should be also attributed to the high intrinsic $k$ of graphene and the effective thermal conductive networks forming by graphene and Ni nanoparticles. The synergistic effects including the stronger phonon Umklapp scattering, better phonon transmission trough the interfaces, decreasing Kapitza resistance, and decreasing ability of heat transfer by electrons result in the slight variation of $k$ with the temperature. The mechanism of thermal transport in the studied composites includes two parts as reported by Balandin et al. $[9,10]$. In their report it shows that the heat in graphene is mostly transferred by acoustic phonons while in silver particles it is transferred by electrons [11]. There are many factors to affect thermal transport ability of composites with changing temperatures. The weak temperature dependence of $k$ is beneficial for TIM applications and can be obtained by controlling the addition of hybrid thermal fillers and quantity of decorated silver nanoparticles.

\section{References}

[1] Condens. Matter Mater. Phys., vol. 79, p. 195425, 2009.

[2] P. A. Khomyakov, G. Giovannetti, P. C. Rusu, G. Brocks, J. Van den Brink and P. J. Kelly, Phys. Rev. B: Condens. Matter Mater. Phys., vol. 79, p. 195425, 2009.

[3] Q. J. Wang and J. G. Che, Phys. Rev. Lett., vol. 103, p. 066802, 2009.

[4] I. Cabria, M. J. L’opez and J. A. Alonso, Phys. Rev. B: Condens. Matter Mater. Phys., vol. 81, p. $035403,2010$.

[5] X. Huang, Z. Y. Yin, S. X. Wu, X. Y. Qi, Q. Y. He, Q. C. Zhang, Q. Y. Yan, F. Boey, and H. Zhang, Small 7, 1876, 2011. 
[6] L. Shang, T. Bian, B. H. Zhang, D. H. Zhang, L. Z. Wu, C. H. Tung, Y. D. Yin, and T. R. Zhang, Angew. Chem. Int. Ed., vol. 53, p. 250, 2014.

[7] Y. C. Si and E. T. Samulski, Chem. Mater., vol. 20, p. 6792, 2008.

[8] Y. Lin, K. A. Watson, M. J. Fallbach, S. Ghose, J. G. Smith Jr., D. M. Delozier, W. Cao, R. E. Crooks, and J. W. Connell. ACS Nano 3, p. 871, 2009.

[9] Z. H. Li, D. Wang, M. Zhang, L. Zhao. Phys. Status. Solidi A., vol. 211, 2142, 2014.

[10] A. A. Balandin, Nature Mat, vol. 10, p. 569, 2011.

[11] D. L. Nika, E. P. Pokatilov, A. S. Askerov, and A. A. Balandin, Phys. Rev. B, vol. 79, p. 155413, 2009. 